\title{
On the Solutions and Conservation Laws of a Coupled Kadomtsev-Petviashvili Equation
}

\author{
Chaudry Masood Khalique \\ Department of Mathematical Sciences, International Institute for Symmetry Analysis and Mathematical Modelling, \\ North-West University, Mafikeng Campus, Private Bag X 2046, Mmabatho 2735, South Africa
}

Correspondence should be addressed to Chaudry Masood Khalique; masood.khalique@nwu.ac.za

Received 6 October 2012; Accepted 2 December 2012

Academic Editor: Asghar Qadir

Copyright (C) 2013 Chaudry Masood Khalique. This is an open access article distributed under the Creative Commons Attribution License, which permits unrestricted use, distribution, and reproduction in any medium, provided the original work is properly cited.

A coupled Kadomtsev-Petviashvili equation, which arises in various problems in many scientific applications, is studied. Exact solutions are obtained using the simplest equation method. The solutions obtained are travelling wave solutions. In addition, we also derive the conservation laws for the coupled Kadomtsev-Petviashvili equation.

\section{Introduction}

The well-known Korteweg-de Vries (KdV) equation [1]

$$
u_{t}+6 u u_{x}+u_{x x x}=0
$$

governs the dynamics of solitary waves. Firstly, it was derived to describe shallow water waves of long wavelength and small amplitude. It is a crucial equation in the theory of integrable systems because it has infinite number of conservation laws, gives multiple-soliton solutions, and has many other physical properties. See, for example, [2] and references therein.

An essential extension of the $\mathrm{KdV}$ equation is the Kadomtsev-Petviashvili (KP) equation given by [3]

$$
\left(u_{t}+6 u u_{x}+u_{x x x}\right)_{x}+u_{y y}=0 .
$$

This equation models shallow long waves in the $x$-direction with some mild dispersion in the $y$-direction. The inverse scattering transform method can be used to prove the complete integrability of this equation. This equation gives multiple-soliton solutions.

Recently, the coupled Korteweg-de Vries equations and the coupled Kadomtsev-Petviashvili equations, because of their applications in many scientific fields, have been the focus of attention for scientists and as a result many studies have been conducted [4-9].
In this paper, we study a new coupled KP equation [10]:

$$
\begin{aligned}
& \left(u_{t}+u_{x x x}-\frac{7}{4} u u_{x}-v v_{x}+\frac{5}{4}(u v)_{x}\right)_{x}+u_{y y}=0, \\
& \left(v_{t}+v_{x x x}-\frac{5}{4} u u_{x}-\frac{7}{4} v v_{x}+2(u v)_{x}\right)_{x}+v_{y y}=0,
\end{aligned}
$$

and find exact solutions of this equation. The method that is employed to obtain the exact solutions for the coupled Kadomtsev-Petviashvili equation ((3a) and (3b)) is the simplest equation method $[11,12]$. Secondly, we derive conservation laws for the system ((3a) and (3b)) using the multiplier approach $[13,14]$.

The simplest equation method was introduced by Kudryashov [11] and later modified by Vitanov [12]. The simplest equations that are used in this method are the Bernoulli and Riccati equations. This method provides a very effective and powerful mathematical tool for solving nonlinear equations in mathematical physics.

Conservation laws play a vital role in the solution process of differential equations (DEs). The existence of a large number of conservation laws of a system of partial differential equations (PDEs) is a strong indication of its integrability [15]. A conserved quantity was utilized to find the unknown exponent in the similarity solution which could not have been obtained from the homogeneous boundary conditions [16]. 
Also recently, conservation laws have been employed to find solutions of the certain PDEs [17-19].

The outline of the paper is as follows. In Section 2, we obtain exact solutions of the coupled KP system ((3a) and (3b)) using the simplest equation method. Conservation laws for ((3a) and (3b)) using the multiplier method are derived in Section 3. Finally, in Section 4 concluding remarks are presented.

\section{Exact Solutions of ((3a) and (3b)) Using Simplest Equation Method}

We first transform the system of partial differential equations ((3a) and (3b)) into a system of nonlinear ordinary differential equations in order to derive its exact solutions.

The transformation

$$
u=F(z), \quad v=G(z), \quad z=t-\rho x+(\rho-1) y,
$$

where $\rho$ is a real constant, transforms $((3 a)$ and $(3 b))$ to the following nonlinear coupled ordinary differential equations (ODEs):

$$
\begin{aligned}
\rho^{4} F^{\prime \prime \prime \prime} & (z)+\frac{5}{4} \rho^{2} G(z) F^{\prime \prime}(z) \\
- & \frac{7}{4} \rho^{2} F(z) F^{\prime \prime}(z)+\rho^{2} F^{\prime \prime}(z) \\
- & 3 \rho F^{\prime \prime}(z)+F^{\prime \prime}(z)+\frac{5}{2} \rho^{2} F^{\prime}(z) G^{\prime}(z) \\
& -\frac{7}{4} \rho^{2} F^{\prime}(z)^{2}+\frac{5}{4} \rho^{2} F(z) G^{\prime \prime}(z) \\
& -\rho^{2} G(z) G^{\prime \prime}(z)-\rho^{2} G^{\prime}(z)^{2}=0, \\
\rho^{4} G^{\prime \prime \prime \prime} & (z)+2 \rho^{2} G(z) F^{\prime \prime}(z) \\
- & \frac{5}{4} \rho^{2} F(z) F^{\prime \prime}(z)+4 \rho^{2} F^{\prime}(z) G^{\prime}(z) \\
& -\frac{5}{4} \rho^{2} F^{\prime}(z)^{2}+2 \rho^{2} F(z) G^{\prime \prime}(z) \\
& -\frac{7}{4} \rho^{2} G(z) G^{\prime \prime}(z)+\rho^{2} G^{\prime \prime}(z) \\
& -3 \rho G^{\prime \prime}(z)+G^{\prime \prime}(z)-\frac{7}{4} \rho^{2} G^{\prime}(z)^{2}=0 .
\end{aligned}
$$

We now use the simplest equation method $[11,12]$ to solve the system $((5 a)$ and $(5 b))$ and as a result we will obtain the exact solutions of our coupled KP system ((3a) and (3b)). We use the Bernoulli and Riccati equations as the simplest equations.

We briefly recall the simplest equation method here. Let us consider the solutions of $((5 a)$ and $(5 b))$ in the form

$$
\begin{aligned}
& F(z)=\sum_{i=0}^{M} \mathscr{A}_{i}(H(z))^{i}, \\
& G(z)=\sum_{i=0}^{M} \mathscr{B}_{i}(H(z))^{i} .
\end{aligned}
$$

Here $H(z)$ satisfies the Bernoulli and Riccati equations, $M$ is a positive integer that can be determined by balancing procedure, and $\mathscr{A}_{0}, \ldots, \mathscr{A}_{M}, \mathscr{B}_{0}, \ldots, \mathscr{B}_{M}$ are constants to be determined. The solutions of the Bernoulli and Riccati equations can be expressed in terms of elementary functions.

We first consider the Bernoulli equation:

$$
H^{\prime}(z)=a H(z)+b H^{2}(z)
$$

where $a$ and $b$ are constants. Its solution can be written as

$$
H(z)=a\left\{\frac{\cosh [a(z+C)]+\sinh [a(z+C)]}{1-b \cosh [a(z+C)]-b \sinh [a(z+C)]}\right\} .
$$

Secondly, for the Riccati equation:

$$
H^{\prime}(z)=a H^{2}(z)+b H(z)+c
$$

( $a, b$, and $c$ are constants), we shall use the solutions

$$
\begin{aligned}
H(z)= & -\frac{b}{2 a}-\frac{\theta}{2 a} \tanh \left[\frac{1}{2} \theta(z+C)\right], \\
H(z)= & -\frac{b}{2 a}-\frac{\theta}{2 a} \tanh \left(\frac{1}{2} \theta z\right) \\
& +\frac{\operatorname{sech}(\theta z / 2)}{C \cosh (\theta z / 2)-(2 a / \theta) \sinh (\theta z / 2)},
\end{aligned}
$$

where $\theta^{2}=b^{2}-4 a c>0$ and $C$ is a constant of integration.

2.1. Solutions of ((3a) and (3b)) Using the Bernoulli Equation as the Simplest Equation. In this case the balancing procedure yields $M=2$ so the solutions of ((5a) and (5b)) are of the form

$$
\begin{aligned}
& F(z)=\mathscr{A}_{0}+\mathscr{A}_{1} H+\mathscr{A}_{2} H^{2}, \\
& G(z)=\mathscr{B}_{0}+\mathscr{B}_{1} H+\mathscr{B}_{2} H^{2} .
\end{aligned}
$$

Substituting (11) into ((5a) and (5b)) and making use of the Bernoulli equation (7) and then equating all coefficients of the functions $H^{i}$ to zero, we obtain an algebraic system of equations in terms of $\mathscr{A}_{0}, \mathscr{A}_{1}, \mathscr{A}_{2}, \mathscr{B}_{0}, \mathscr{B}_{1}$, and $\mathscr{B}_{2}$. 
Solving the system of algebraic equations, with the aid of Mathematica, we obtain

$$
\begin{aligned}
& a=1, \quad b=3, \\
& \mathscr{A}_{0}=\frac{k\left(\rho^{4}+\rho^{2}-3 \rho+1\right)}{\rho^{2}}, \\
& \mathscr{A}_{1}=\frac{36 \mathscr{A}_{0} \rho^{4}}{\rho^{4}+\rho^{2}-3 \rho+1}, \\
& \mathscr{A}_{2}=3 \mathscr{A}_{1} \text {, } \\
& \mathscr{B}_{0}=\frac{1}{21312} \\
& \times\left\{-49248 \mathscr{A}_{0} \rho^{9}-8208 \mathscr{A}_{0} \rho^{8}\right. \\
& +67392 \mathscr{A}_{0}^{2} \rho^{7}-98496 \mathscr{A}_{0} \rho^{7} \\
& +11232 \mathscr{A}_{0}^{2} \rho^{6}+279072 \mathscr{A}_{0} \rho^{6} \\
& -75978 \mathscr{A}_{0}^{3} \rho^{5}+67392 \mathscr{A}_{0}^{2} \rho^{5} \\
& -98496 \mathscr{A}_{0} \rho^{5}-12663 \mathscr{A}_{0}^{3} \rho^{4} \\
& -190944 \mathscr{A}_{0}^{2} \rho^{4}+270864 \mathscr{A}_{0} \rho^{4} \\
& -67608 \mathscr{A}_{0}^{2} \rho^{3}-492480 \mathscr{A}_{0} \rho^{3} \\
& +2814 \mathscr{A}_{0} \mathscr{A}_{1} \rho^{3}-22536 \mathscr{A}_{0}^{2} \rho^{2} \\
& +205200 \mathscr{A}_{0} \rho^{2}+938 \mathscr{A}_{0} \mathscr{A}_{1} \rho^{2} \\
& +2814 \mathscr{A}_{0} \mathscr{A}_{1} \rho+34704 \mathscr{A}_{0} \\
& -7504 \mathscr{A}_{0} \mathscr{A}_{1}+41472 \rho^{11} \\
& +6912 \rho^{10}+124416 \rho^{9} \\
& -352512 \rho^{8}+186624 \rho^{7} \\
& -705024 \rho^{6}+1285632 \rho^{5} \\
& -884736 \rho^{4}+1181952 \rho^{3} \\
& \left.-1645056 \rho^{2}+933120 \rho-172800\right\}, \\
& \mathscr{B}_{1}=\frac{\mathscr{A}_{1}}{1022976} \\
& \times\left\{9849 \mathscr{A}_{1}^{2} \rho^{3}-3245184 \mathscr{A}_{0} \rho^{3}\right. \\
& -354564 \mathscr{A}_{0} \mathscr{A}_{1} \rho^{3}+90144 \mathscr{A}_{1} \rho^{3} \\
& +3752 \mathscr{A}_{1}^{2} \rho^{2}-1081728 \mathscr{A}_{0} \rho^{2} \\
& -135072 \mathscr{A}_{0} \mathscr{A}_{1} \rho^{2}+30048 \mathscr{A}_{1} \rho^{2} \\
& +11256 \mathscr{A}_{1}^{2} \rho-50652 \mathscr{A}_{0} \mathscr{A}_{1} \rho \\
& +90144 \mathscr{A}_{1} \rho-25326 \mathscr{A}_{1}^{2} \\
& \left.-16884 \mathscr{A}_{0} \mathscr{A}_{1}-240384 \mathscr{A}_{1}+1665792\right\} \text {, }
\end{aligned}
$$

$$
\begin{aligned}
& \mathscr{B}_{2}=\frac{\mathscr{A}_{1}}{340992} \\
& \times\left\{9849 \mathscr{A}_{1}^{2} \rho^{3}\right. \\
& \quad-3245184 \mathscr{A}_{0} \rho^{3}-354564 \mathscr{A}_{0} \mathscr{A}_{1} \rho^{3} \\
&+ 90144 \mathscr{A}_{1} \rho^{3}+3752 \mathscr{A}_{1}^{2} \rho^{2} \\
&-1081728 \mathscr{A}_{0} \rho^{2}-135072 \mathscr{A}_{0} \mathscr{A}_{1} \rho^{2} \\
&+30048 \mathscr{A}_{1} \rho^{2}+11256 \mathscr{A}_{1}^{2} \rho \\
&-50652 \mathscr{A}_{0} \mathscr{A}_{1} \rho+90144 \mathscr{A}_{1} \rho \\
&-25326 \mathscr{A}_{1}^{2}-16884 \mathscr{A}_{0} \mathscr{A}_{1} \\
&\left.-240384 \mathscr{A}_{1}+1665792\right\}
\end{aligned}
$$

where $k$ is any root of $469 k^{3}-416 k^{2}+304 k-256=0$. Consequently, a solution of $((3 a)$ and $(3 b))$ is given by

$$
\begin{aligned}
& u(t, x, y) \\
& =A_{0}+A_{1} a\left\{\frac{\cosh [a(z+C)]+\sinh [a(z+C)]}{1-b \cosh [a(z+C)]-b \sinh [a(z+C)]}\right\} \\
& +A_{2} a^{2}\left\{\frac{\cosh [a(z+C)]+\sinh [a(z+C)]}{1-b \cosh [a(z+C)]-b \sinh [a(z+C)]}\right\}^{2}, \\
& v(t, x, y) \\
& =B_{0}+B_{1} a\left\{\frac{\cosh [a(z+C)]+\sinh [a(z+C)]}{1-b \cosh [a(z+C)]-b \sinh [a(z+C)]}\right\} \\
& +B_{2} a^{2}\left\{\frac{\cosh [a(z+C)]+\sinh [a(z+C)]}{1-b \cosh [a(z+C)]-b \sinh [a(z+C)]}\right\}^{2},
\end{aligned}
$$

where $z=t-\rho x+(\rho-1) y$ and $C$ is a constant of integration.

2.2. Solutions of ((3a) and (3b)) Using Riccati Equation as the Simplest Equation. The balancing procedure gives $M=2$ so the solutions of $((5 a)$ and $(5 b))$ are of the form

$$
\begin{aligned}
& F(z)=\mathscr{A}_{0}+\mathscr{A}_{1} H+\mathscr{A}_{2} H^{2}, \\
& G(z)=\mathscr{B}_{0}+\mathscr{B}_{1} H+\mathscr{B}_{2} H^{2} .
\end{aligned}
$$

Substituting (14) into ((5a) and (5b)) and making use of the Riccati equation (9), we obtain algebraic system of equations in terms of $\mathscr{A}_{0}, \mathscr{A}_{1}, \mathscr{A}_{2}, \mathscr{B}_{0}, \mathscr{B}_{1}$, and $\mathscr{B}_{2}$ by equating all coefficients of the functions $H^{i}$ to zero. 
Solving the algebraic equations one obtains

$$
\begin{aligned}
& \rho=-1 \\
& \mathscr{A}_{0}=k\left(8 a c+b^{2}+5\right) \text {, } \\
& \mathscr{A}_{1}=\frac{12 a \mathscr{A}_{0} b}{8 a c+b^{2}+5}, \\
& \mathscr{A}_{2}=\frac{a \mathscr{A}_{1}}{b} \text {, } \\
& \mathscr{B}_{0}=3\left(-2048 a^{2} b c-256 a b^{3}+208 a \mathscr{A}_{0} b\right. \\
& \left.-1280 a b+15 \mathscr{A}_{0} \mathscr{A}_{1}\right) \times\left(74 \mathscr{A}_{1}\right)^{-1}, \\
& \mathscr{B}_{1}=\frac{\mathscr{A}_{1}\left(336 a b-29 \mathscr{A}_{1}\right)}{192 a b-6 \mathscr{A}_{1}} \text {, } \\
& \mathscr{B}_{2}=\frac{1}{17760000} \\
& \times\left\{-270144 a^{2} \mathscr{A}_{0}^{2} \mathscr{A}_{1} b c\right. \\
& +1080576 a^{2} \mathscr{A}_{0}^{2} \mathscr{A}_{2} c^{2}+50652 a \mathscr{A}_{0}^{2} \mathscr{A}_{1} b^{3} \\
& \text { - 84420a } \mathscr{A}_{0}^{2} \mathscr{A}_{1} b+5784000 a \mathscr{A}_{1} b \\
& \text { - 751200a } \mathscr{A}_{0} \mathscr{A}_{1} b-3552000 a b \mathscr{B}_{1} \\
& +1350720 a \mathscr{A}_{0}^{2} \mathscr{A}_{2} c+6009600 a \mathscr{A}_{0} \mathscr{A}_{2} c \\
& +70350 \mathscr{A}_{0} \mathscr{A}_{1}^{2}+313000 \mathscr{A}_{1}^{2} \\
& -422100 \mathscr{A}_{0}^{2} \mathscr{A}_{2}-3756000 \mathscr{A}_{0} \mathscr{A}_{2} \\
& +28920000 \mathscr{A}_{2}-4221 \mathscr{A}_{0} \mathscr{A}_{1}^{2} b^{4} \\
& -938 \mathscr{A}_{1}^{3} b^{3} c-14070 \mathscr{A}_{0} \mathscr{A}_{1}^{2} b^{2} \\
& +62600 \mathscr{A}_{1}^{2} b^{2}-480256 \mathscr{A}_{2}^{3} c^{4} \\
& -4006400 \mathscr{A}_{2}^{2} c^{2}-1800960 \mathscr{A}_{0} \mathscr{A}_{2}^{2} c^{2} \\
& \left.+112560 \mathscr{A}_{1}^{2} \mathscr{A}_{2} c^{2}\right\} \text {, }
\end{aligned}
$$

where $k$ is any root of $469 k^{3}-416 k^{2}+304 k-256$ and hence solutions of ((3a) and (3b)) are

$$
\begin{aligned}
u(t, x, y)= & A_{0}+A_{1}\left\{-\frac{b}{2 a}-\frac{\theta}{2 a} \tanh \left[\frac{1}{2} \theta(z+C)\right]\right\} \\
& +A_{2}\left\{-\frac{b}{2 a}-\frac{\theta}{2 a} \tanh \left[\frac{1}{2} \theta(z+C)\right]\right\}^{2} \\
v(t, x, y)= & B_{0}+B_{1}\left\{-\frac{b}{2 a}-\frac{\theta}{2 a} \tanh \left[\frac{1}{2} \theta(z+C)\right]\right\} \\
& +B_{2}\left\{-\frac{b}{2 a}-\frac{\theta}{2 a} \tanh \left[\frac{1}{2} \theta(z+C)\right]\right\}^{2}
\end{aligned}
$$

$$
\begin{aligned}
& u(t, x)=A_{0}+A_{1}\left\{-\frac{b}{2 a}-\frac{\theta}{2 a} \tanh \left(\frac{1}{2} \theta z\right)\right. \\
& \left.+\frac{\operatorname{sech}(\theta z / 2)}{C \cosh (\theta z / 2)-(2 a / \theta) \sinh (\theta z / 2)}\right\} \\
& +A_{2}\left\{-\frac{b}{2 a}-\frac{\theta}{2 a} \tanh \left(\frac{1}{2} \theta z\right)\right. \\
& \left.+\frac{\operatorname{sech}(\theta z / 2)}{C \cosh (\theta z / 2)-(2 a / \theta) \sinh (\theta z / 2)}\right\}^{2}, \\
& v(t, x)=B_{0}+B_{1}\left\{-\frac{b}{2 a}-\frac{\theta}{2 a} \tanh \left(\frac{1}{2} \theta z\right)\right. \\
& \left.+\frac{\operatorname{sech}(\theta z / 2)}{C \cosh (\theta z / 2)-(2 a / \theta) \sinh (\theta z / 2)}\right\} \\
& +B_{2}\left\{-\frac{b}{2 a}-\frac{\theta}{2 a} \tanh \left(\frac{1}{2} \theta z\right)\right. \\
& \left.+\frac{\operatorname{sech}(\theta z / 2)}{C \cosh (\theta z / 2)-(2 a / \theta) \sinh (\theta z / 2)}\right\}^{2} \text {, }
\end{aligned}
$$

where $z=t-p x+(p-1) y$ and $C$ is a constant of integration.

A profile of the solution $((13 a)$ and $(13 b))$ is given in Figure 1 . The flat peaks appearing in the figure are an artifact of Mathematica and they describe the singularities of the solution.

\section{Conservation Laws of ((3a) and (3b))}

In this section we present conservation laws for the coupled KP system ((3a) and (3b)) using the multiplier method [13, 14]. First we present some preliminaries which we will need later in this section.

3.1. Preliminaries. We briefly present the notation and pertinent results which we utilize below. For details the reader is referred to [20].

Consider a $k$ th-order system of PDEs of $n$-independent variables $x=\left(x^{1}, x^{2}, \ldots, x^{n}\right)$ and $m$-dependent variables $u=$ $\left(u^{1}, u^{2}, \ldots, u^{m}\right)$ :

$$
E_{\alpha}\left(x, u, u_{(1)}, \ldots, u_{(k)}\right)=0, \quad \alpha=1, \ldots, m,
$$

where $u_{(1)}, u_{(2)}, \ldots, u_{(k)}$ denote the collections of all first, second,..., $k$ th-order partial derivatives, that is, $u_{i}^{\alpha}=$ $D_{i}\left(u^{\alpha}\right), u_{i j}^{\alpha}=D_{j} D_{i}\left(u^{\alpha}\right), \ldots$, respectively, with the total derivative operator with respect to $x^{i}$ given by

$$
D_{i}=\frac{\partial}{\partial x^{i}}+u_{i}^{\alpha} \frac{\partial}{\partial u^{\alpha}}+u_{i j}^{\alpha} \frac{\partial}{\partial u_{j}^{\alpha}}+\cdots, \quad i=1, \ldots, n,
$$

where the summation convention is used whenever appropriate. 


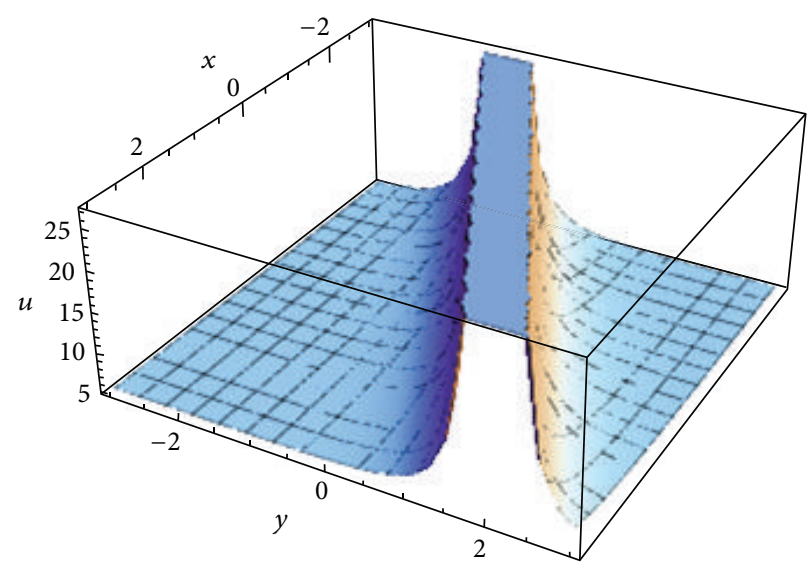

(a)

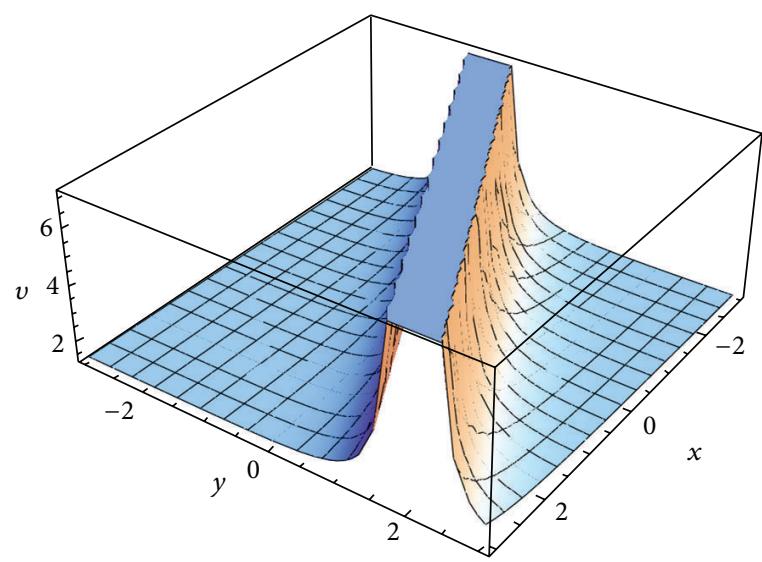

(b)

FIGURE 1: Profile of the travelling wave solution ((13a) and (13b)).

The Euler-Lagrange operator, for each $\alpha$, is given by

$$
\begin{array}{r}
\frac{\delta}{\delta u^{\alpha}}=\frac{\partial}{\partial u^{\alpha}}+\sum_{s \geq 1}(-1)^{s} D_{i_{1}}, \ldots, D_{i_{s}} \frac{\partial}{\partial u_{i_{1} i_{2}, \ldots, i_{s}}^{\alpha}} \\
\alpha=1, \ldots, m .
\end{array}
$$

The $n$-tuple vector $T=\left(T^{1}, T^{2}, \ldots, T^{n}\right), T^{j} \in \mathscr{A}, j=$ $1, \ldots, n$, where $\mathscr{A}$ is the space of differential functions, is a conserved vector of (18) if $T^{i}$ satisfies

$$
\left.D_{i} T^{i}\right|_{(18)}=0
$$

Equation (21) defines a local conservation law of system (18). A multiplier $\Lambda_{\alpha}\left(x, u, u_{(1)}, \ldots\right)$ has the property that

$$
\Lambda_{\alpha} E_{\alpha}=D_{i} T^{i}
$$

holds identically. In this paper, we will consider multipliers of the zeroth order, that is, $\Lambda_{\alpha}=\Lambda_{\alpha}(t, x, y, u, v)$. The determining equations for the multiplier $\Lambda_{\alpha}$ are

$$
\frac{\delta\left(\Lambda_{\alpha} E_{\alpha}\right)}{\delta u^{\alpha}}=0
$$

Once the multipliers are obtained the conserved vectors are calculated via a homotopy formula $[13,14]$.

3.2. Construction of Conservation Laws for ((3a) and (3b)). We now construct conservation laws for the coupled KP system ((3a) and (3b)) using the multiplier method. For the coupled KP system ((3a) and (3b)), we obtain the zerothorder multipliers (with the aid of GeM [21]), $\Lambda_{1}(t, x, y, u, v)$, $\Lambda_{2}(t, x, y, u, v)$ that are given by

$$
\begin{aligned}
\Lambda_{1}= & f_{3}(t)+y f_{4}(t)-y^{2} f_{7}^{\prime}(t)+2 x f_{7}(t) \\
& +y^{3}\left(-f_{8}^{\prime}(t)\right)+6 x y f_{8}(t), \\
\Lambda_{2}= & -y^{2} f_{1}^{\prime}(t)+2 x f_{1}(t)+y^{3}\left(-f_{2}^{\prime}(t)\right) \\
& +6 x y f_{2}(t)+f_{5}(t)+y f_{6}(t),
\end{aligned}
$$

where $f_{i}, i=1,2, \ldots, 8$ are arbitrary functions of $t$.

Corresponding to the above multipliers we have the following eight local conserved vectors of ((3a) and (3b)):

$$
\begin{aligned}
T_{1}^{t}=\frac{1}{2}\{ & \left.-2 f_{1}(t) v+2 x f_{1}(t) v_{x}-y^{2} f_{1}^{\prime}(t) v_{x}\right\}, \\
T_{1}^{x}=\frac{1}{4}\{ & -8 y^{2} f_{1}^{\prime}(t) u_{x} v-8 y^{2} f_{1}^{\prime}(t) v_{x} u \\
& +16 x f_{1}(t) u_{x} v+16 x f_{1}(t) v_{x} u \\
& +5 y^{2} f_{1}^{\prime}(t) u_{x} u-10 x f_{1}(t) u_{x} u \\
& +7 y^{2} f_{1}^{\prime}(t) v_{x} v-14 x f_{1}(t) v_{x} v \\
& -16 f_{1}(t) u v+5 f_{1}(t) u^{2}+2 y^{2} f_{1}^{\prime \prime}(t) v \\
& -4 x f_{1}^{\prime}(t) v+7 f_{1}(t) v^{2}-8 f_{1}(t) v_{x x} \\
& +8 x f_{1}(t) v_{x x x}+4 x f_{1}(t) v_{t} \\
& \left.-2 y^{2} f_{1}^{\prime}(t) v_{t}-4 y^{2} f_{1}^{\prime}(t) v_{x x x}\right\},
\end{aligned}
$$




$$
\begin{aligned}
& T_{1}^{y}=2 y f_{1}^{\prime}(t) v+2 x f_{1}(t) v_{y}-y^{2} f_{1}^{\prime}(t) v_{y}, \\
& T_{2}^{t}=\frac{1}{2}\left\{-6 y f_{2}(t) v+6 x y f_{2}(t) v_{x}+y^{3}\left(-f_{2}^{\prime}(t)\right) v_{x}\right\} \text {, } \\
& T_{2}^{x}=\frac{1}{4}\left\{-8 y^{3} f_{2}^{\prime}(t) u_{x} v-8 y^{3} f_{2}^{\prime}(t) v_{x} u\right. \\
& +48 x y f_{2}(t) u_{x} v+48 x y f_{2}(t) v_{x} u \\
& +5 y^{3} f_{2}^{\prime}(t) u_{x} u-30 x y f_{2}(t) u_{x} u \\
& +7 y^{3} f_{2}^{\prime}(t) v_{x} v-42 x y f_{2}(t) v_{x} v \\
& -48 y f_{2}(t) u v+15 y f_{2}(t) u^{2} \\
& +2 y^{3} f_{2}^{\prime \prime}(t) v-12 x y f_{2}^{\prime}(t) v \\
& +21 y f_{2}(t) v^{2}-24 y f_{2}(t) v_{x x} \\
& +24 x y f_{2}(t) v_{x x x}+12 x y f_{2}(t) v_{t} \\
& \left.-2 y^{3} f_{2}^{\prime}(t) v_{t}-4 y^{3} f_{2}^{\prime}(t) v_{x x x}\right\}, \\
& T_{2}^{y}=3 y^{2} f_{2}^{\prime}(t) v-6 x f_{2}(t) v \\
& +6 x y f_{2}(t) v_{y}-y^{3} f_{2}^{\prime}(t) v_{y}, \\
& T_{3}^{t}=\frac{1}{2} f_{3}(t) u_{x} \\
& T_{3}^{x}=\frac{1}{4}\left\{5 f_{3}(t) u_{x} v+5 f_{3}(t) v_{x} u\right. \\
& -7 f_{3}(t) u_{x} u-4 f_{3}(t) v_{x} v \\
& \left.-2 f_{3}^{\prime}(t) u+4 f_{3}(t) u_{x x x}+2 f_{3}(t) u_{t}\right\} \text {, } \\
& T_{3}^{y}=f_{3}(t) u_{y} \\
& T_{4}^{t}=\frac{1}{2} y f_{4}(t) u_{x} \\
& T_{4}^{x}=\frac{1}{4}\left\{5 y f_{4}(t) u_{x} v+5 y f_{4}(t) v_{x} u\right. \\
& -7 y f_{4}(t) u_{x} u-4 y f_{4}(t) v_{x} v-2 y f_{4}^{\prime} u \\
& \left.+4 y f_{4}(t) u_{x x x}+2 y f_{4}(t) u_{t}\right\} \\
& T_{4}^{y}=y f_{4}(t) u_{y}-f_{4}(t) u, \\
& T_{5}^{t}=\frac{1}{2} f_{5}(t) v_{x} \\
& T_{5}^{x}=\frac{1}{4}\left\{8 f_{5}(t) u_{x} v+8 f_{5}(t) v_{x} u\right. \\
& -5 f_{5}(t) u_{x} u-7 f_{5}(t) v_{x} v-2 f_{5}^{\prime}(t) v \\
& \left.+4 f_{5}(t) v_{x x x}+2 f_{5}(t) v_{t}\right\} \\
& T_{5}^{y}=f_{5}(t) v_{y}, \\
& T_{6}^{t}=\frac{1}{2} y f_{6}(t) v_{x} \\
& T_{6}^{x}=\frac{1}{4}\left\{8 y f_{6}(t) u_{x} v+8 y f_{6}(t) v_{x} u\right. \\
& -5 y f_{6}(t) u_{x} u-7 y f_{6}(t) v_{x} v \\
& -2 y f_{6}^{\prime}(t) v+4 y f_{6}(t) v_{x x x} \\
& \left.+2 y f_{6}(t) v_{t}\right\} \\
& T_{6}^{y}=y f_{6}(t) v_{y}-f_{6}(t) v, \\
& T_{7}^{t}=\frac{1}{2}\left\{-2 f_{7}(t) u+2 x f_{7}(t) u_{x}-y^{2} f_{7}^{\prime}(t) u_{x}\right\}, \\
& T_{7}^{x}=\frac{1}{4}\left\{-5 y^{2} f_{7}^{\prime}(t) u_{x} v-5 y^{2} f_{7}^{\prime}(t) v_{x} u\right. \\
& +10 x f_{7}(t) u_{x} v+10 x f_{7}(t) v_{x} u \\
& +7 y^{2} f_{7}^{\prime}(t) u_{x} u-14 x f_{7}(t) u_{x} u \\
& +4 y^{2} f_{7}^{\prime}(t) v_{x} v-8 x f_{7}(t) v_{x} v \\
& -10 f_{7}(t) u v+2 y^{2} f_{7}^{\prime \prime}(t) u \\
& -4 x f_{7}^{\prime}(t) u+7 f_{7}(t) u^{2} \\
& +4 f_{7}(t) v^{2}-8 f_{7}(t) u_{x x} \\
& +8 x f_{7}(t) u_{x x x}+4 x f_{7}(t) u_{t} \\
& \left.-2 y^{2} f_{7}^{\prime}(t) u_{t}-4 y^{2} f_{7}^{\prime}(t) u_{x x x}\right\}, \\
& T_{7}^{y}=2 y f_{7}^{\prime}(t) u+2 x f_{7}(t) u_{y}-y^{2} f_{7}^{\prime}(t) u_{y} \\
& T_{8}^{t}=\frac{1}{2}\left\{-6 y f_{8}(t) u+6 x y f_{8}(t) u_{x}-y^{3} f_{8}^{\prime}(t) u_{x}\right\}, \\
& T_{8}^{x}=\frac{1}{4}\left\{-5 y^{3} f_{8}^{\prime}(t) u_{x} v-5 y^{3} f_{8}^{\prime}(t) v_{x} u\right. \\
& +30 x y f_{8}(t) u_{x} v+30 x y f_{8}(t) v_{x} u \\
& +7 y^{3} f_{8}^{\prime}(t) u_{x} u-42 x y f_{8}(t) u_{x} u \\
& +4 y^{3} f_{8}^{\prime}(t) v_{x} v-24 x y f_{8}(t) v_{x} v \\
& -30 y f_{8}(t) u v+2 y^{3} f_{8}^{\prime \prime} u \\
& -12 x y f_{8}^{\prime}(t) u+21 y f_{8}(t) u^{2} \\
& +12 y f_{8}(t) v^{2}-24 y f_{8}(t) u_{x x} \\
& +24 x y f_{8}(t) u_{x x x}+12 x y f_{8}(t) u_{t} \\
& \left.-2 y^{3} f_{8}^{\prime}(t) u_{t}-4 y^{3} f_{8}^{\prime}(t) u_{x x x}\right\}, \\
& T_{8}^{y}=3 y^{2} f_{8}^{\prime}(t) u-6 x f_{8}(t) u \\
& +6 x y f_{8}(t) u_{y}-y^{3} f_{8}^{\prime}(t) u_{y} .
\end{aligned}
$$


We note that because of the arbitrary functions $f_{i}, i=$ $1,2, \ldots, 8$ in the multipliers, we obtain an infinitely many conservation laws for the coupled KP system ((3a) and (3b)).

\section{Concluding Remarks}

The coupled Kadomtsev-Petviashvili system ((3a) and (3b)) was studied in this paper. The simplest equation method was used to obtain travelling wave solutions of the coupled $\mathrm{KP}$ system ((3a) and (3b)). The simplest equations that were used in the solution process were the Bernoulli and Riccati equations. However, it should be noted that the solutions $((13 a)$ and $(13 b)),((16 a)$ and $(16 b))$, and ((17a) and (17b)) obtained by using these simplest equations are not connected to each other. We have checked the correctness of the solutions obtained here by substituting them back into the coupled KP system ((3a) and (3b)). Furthermore, infinitely many conservation laws for the coupled KP system ((3a) and (3b)) were derived by employing the multiplier method. The importance of constructing the conservation laws was discussed in the introduction.

\section{Acknowledgments}

C. M. Khalique would like to thank the Organizing Committee of Symmetries, Differential Equations, and Applications: Galois Bicentenary (SDEA2012) Conference for their kind hospitality during the conference.

\section{References}

[1] D. J. Korteweg and G. de Vries, "On the change of form of long waves advancing in a rectangular canal, and on a new type of long stationary waves," Philosophical Magazine, vol. 39, pp. 422443, 1895.

[2] A. M. Wazwaz, "Integrability of coupled KdV equations," Central European Journal of Physics, vol. 9, no. 3, pp. 835-840, 2011.

[3] B. B. Kadomtsev and V. I. Petviashvili, "On the stability of solitary waves in weakly dispersive media," Soviet Physics. Doklady, vol. 15, pp. 539-541, 1970.

[4] D.-S. Wang, "Integrability of a coupled KdV system: Painlevé property, Lax pair and Bäcklund transformation," Applied Mathematics and Computation, vol. 216, no. 4, pp. 1349-1354, 2010.

[5] C.-X. Li, "A hierarchy of coupled Korteweg-de Vries equations and the corresponding finite-dimensional integrable system," Journal of the Physical Society of Japan, vol. 73, no. 2, pp. 327331, 2004.

[6] Z. Qin, "A finite-dimensional integrable system related to a new coupled KdV hierarchy," Physics Letters A, vol. 355, no. 6, pp. 452-459, 2006.

[7] X. Geng, "Algebraic-geometrical solutions of some multidimensional nonlinear evolution equations," Journal of Physics A, vol. 36, no. 9, pp. 2289-2303, 2003.

[8] X. Geng and Y. Ma, "N-solution and its Wronskian form of a (3+1)-dimensional nonlinear evolution equation," Physics Letters A, vol. 369, no. 4, pp. 285-289, 2007.
[9] X. Geng and G. He, "Some new integrable nonlinear evolution equations and Darboux transformation," Journal of Mathematical Physics, vol. 51, no. 3, Article ID 033514, 21 pages, 2010.

[10] A.-M. Wazwaz, "Integrability of two coupled kadomtsev-petviashvili equations," Pramana, vol. 77, no. 2, pp. 233-242, 2011.

[11] N. A. Kudryashov, "Simplest equation method to look for exact solutions of nonlinear differential equations," Chaos, Solitons \& Fractals, vol. 24, no. 5, pp. 1217-1231, 2005.

[12] N. K. Vitanov, "Application of simplest equations of Bernoulli and Riccati kind for obtaining exact traveling-wave solutions for a class of PDEs with polynomial nonlinearity," Communications in Nonlinear Science and Numerical Simulation, vol. 15, no. 8, pp. 2050-2060, 2010.

[13] S. C. Anco and G. Bluman, "Direct construction method for conservation laws of partial differential equations. I. Examples of conservation law classifications," European Journal of Applied Mathematics, vol. 13, no. 5, pp. 545-566, 2002.

[14] M. Anthonyrajah and D. P. Mason, "Conservation laws and invariant solutions in the Fanno model for turbulent compressible flow," Mathematical \& Computational Applications, vol. 15, no. 4, pp. 529-542, 2010.

[15] G. W. Bluman and S. Kumei, Symmetries and Differential Equations, vol. 81 of Applied Mathematical Sciences, Springer, New York, NY, USA, 1989.

[16] R. Naz, F. M. Mahomed, and D. P. Mason, "Comparison of different approaches to conservation laws for some partial differential equations in fluid mechanics," Applied Mathematics and Computation, vol. 205, no. 1, pp. 212-230, 2008.

[17] A. Sjöberg, "Double reduction of PDEs from the association of symmetries with conservation laws with applications," Applied Mathematics and Computation, vol. 184, no. 2, pp. 608-616, 2007.

[18] A. Sjöberg, "On double reductions from symmetries and conservation laws," Nonlinear Analysis: Real World Applications, vol. 10, no. 6, pp. 3472-3477, 2009.

[19] A. H. Bokhari, A. Y. Al-Dweik, F. D. Zaman, A. H. Kara, and F. M. Mahomed, "Generalization of the double reduction theory," Nonlinear Analysis: Real World Applications, vol. 11, no. 5, pp. 3763-3769, 2010.

[20] N. H. Ibragimov, CRC Handbook of Lie Group Analysis of Differential Equations, vol. 1-3, CRC Press, Boca Raton, Fla, USA, 1996.

[21] A. F. Cheviakov, "GeM software package for computation of symmetries and conservation laws of differential equations," Computer Physics Communications, vol. 176, no. 1, pp. 48-61, 2007. 


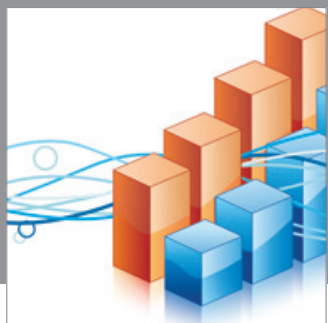

Advances in

Operations Research

mansans

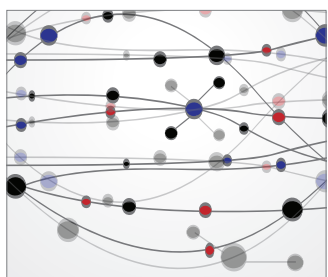

The Scientific World Journal
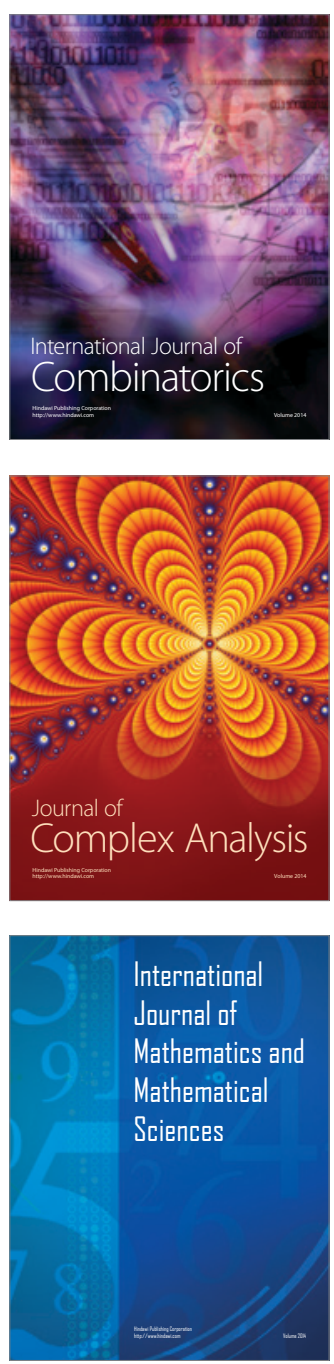
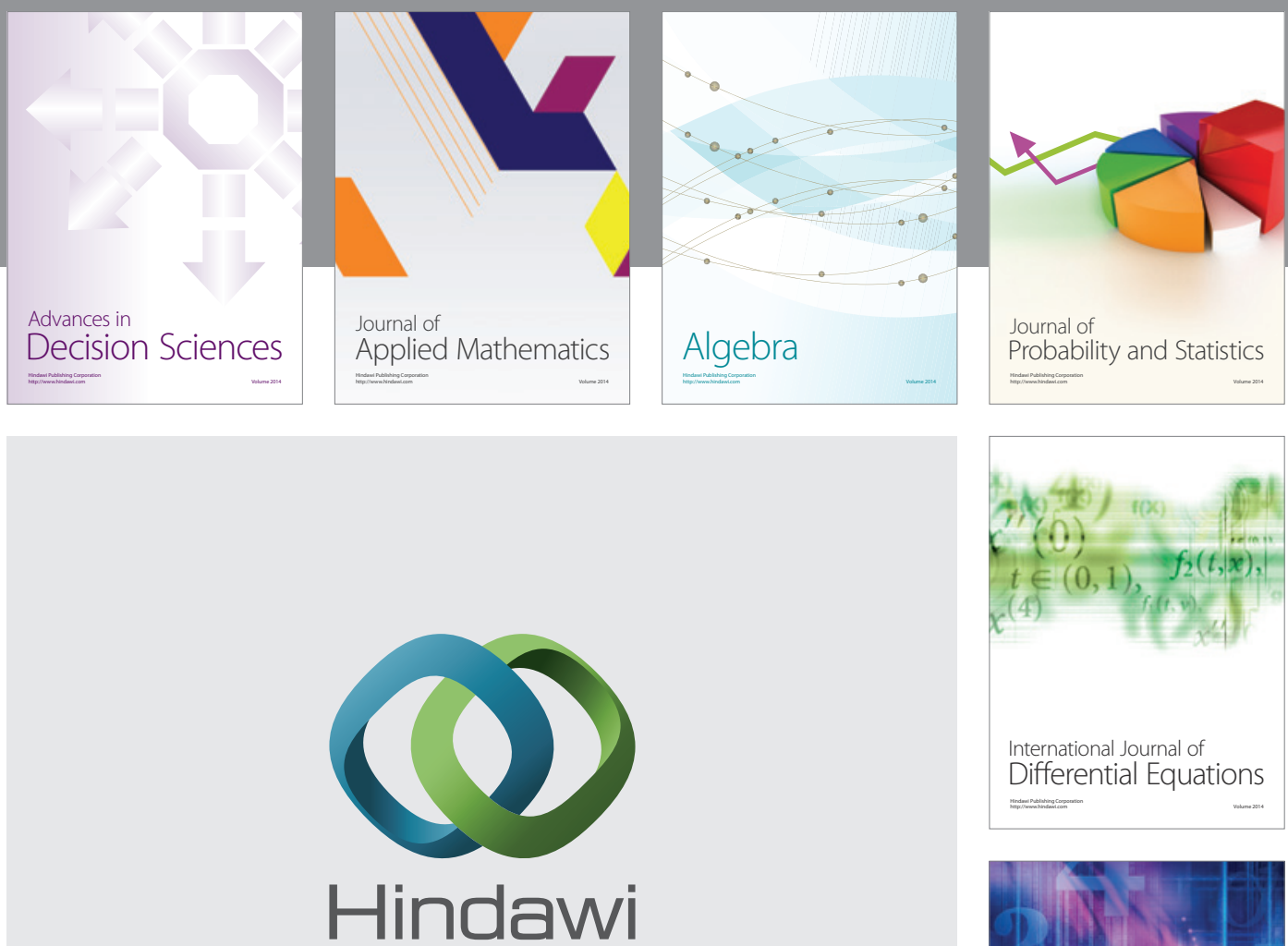

Submit your manuscripts at http://www.hindawi.com
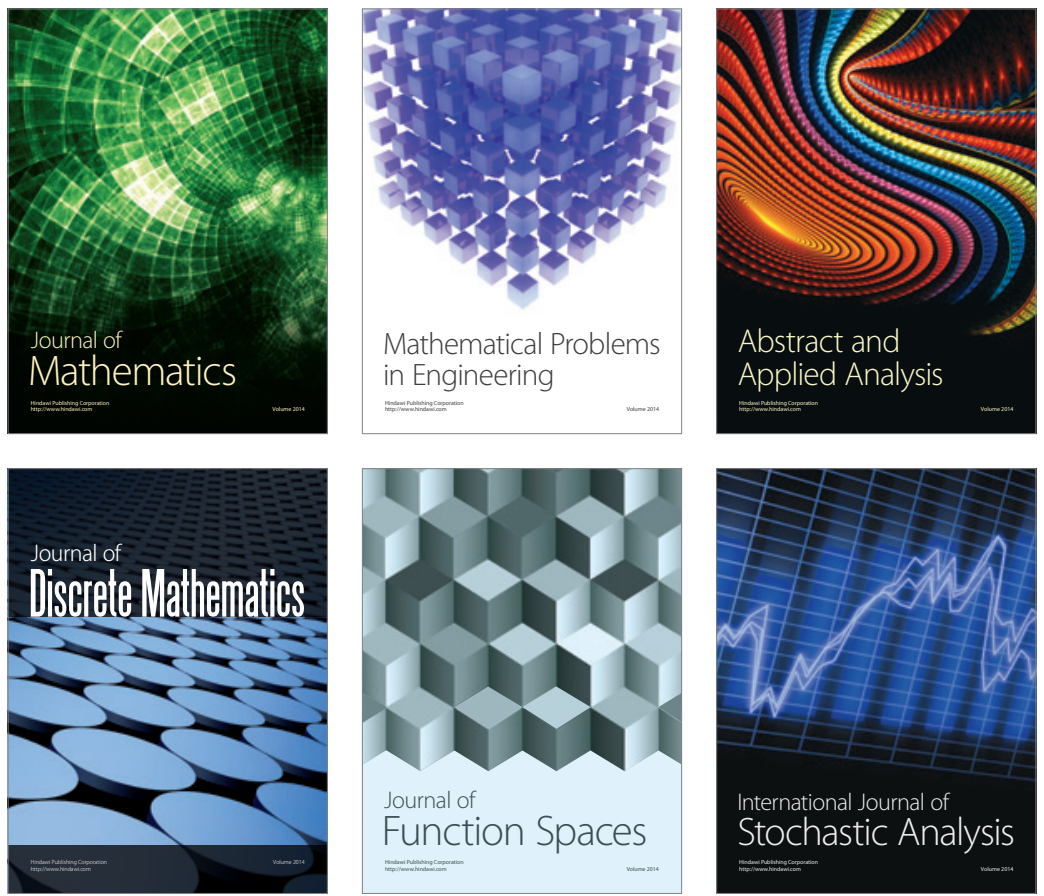

Journal of

Function Spaces

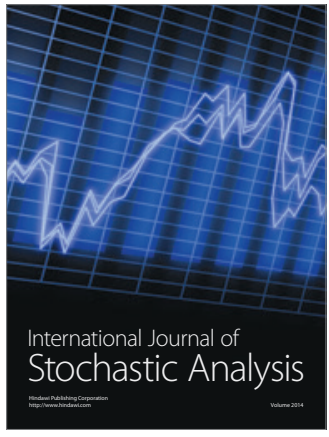

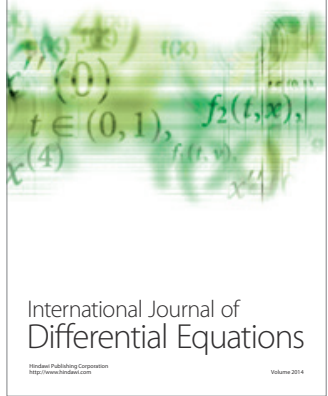
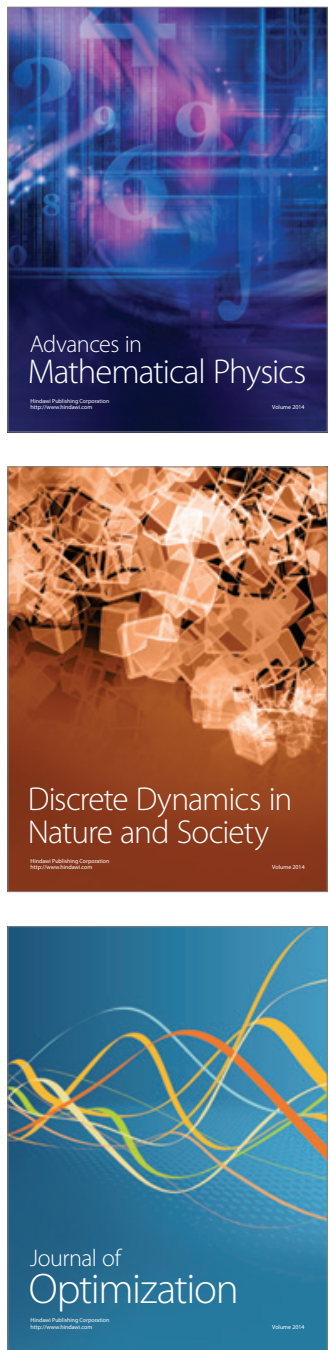